\title{
Altered EEG Brain Networks in Patients with Acute Peripheral Herpes Zoster
}

\author{
Yan Zhou ${ }^{1-3}$ \\ Zhenqin Liu $^{4}$ \\ Yuanmei Sun ${ }^{5}$ \\ Hao Zhang ${ }^{1,3}$ \\ Jianghai Ruan (1) ${ }^{1,3}$ \\ 'Department of Neurology, The Affiliated \\ Hospital of Southwest Medical University, \\ Luzhou, 646000, People's Republic of \\ China; ${ }^{2}$ Department of Neurology, \\ Jianyang People's Hospital, Jianyang, \\ 64I400, People's Republic of China; \\ ${ }^{3}$ Laboratory of Neurological Diseases \\ and Brain Function, Luzhou, 646000 , \\ People's Republic of China; ${ }^{4}$ Department \\ of Dermatology, Jianyang People's \\ Hospital, Jianyang, 64I400, People's \\ Republic of China; ${ }^{5}$ Department of \\ Dermatology, Chongqing Hospital of \\ Traditional Chinese Medicine, Chongqing, \\ 4000 10, People's Republic of China
}

Correspondence: Jianghai Ruan Department of Neurology, The Affiliated Hospital of Southwest Medical University, Taiping Street No. 25, Luzhou, 646000 People's Republic of China

Email jianghai.ruan@swmu.edu.cn
Objective: To investigate whether the brain networks changed in patients with acute peripheral herpes zoster (HZ).

Methods: We reviewed the EEG database in Jianyang People's Hospital. Patients with acute HZ ( $n=71)$ were enrolled from January 2016 to December 2020. Each included subject underwent a ten-minute and 16-channel EEG examination. Five epochs of 10-second EEG data in resting-state were collected from each $\mathrm{HZ}$ patient. Five 10-second resting-state EEG epochs from sex- and age-matched healthy controls ( $\mathrm{HC}, \mathrm{n}=71)$ who reported no history of neurological or psychiatric disorders and visited the hospital for routine physical examinations were collected. Brain network and graph theory analysis based on phase locking value parameter and functional ICA were performed using a self-writing Matlab code and the LORETA KEY tool.

Results: Compared with the HC group, the HZ patients showed significant altered brain networks. The graph theory analysis revealed that the clustering coefficient and local efficiency of full band in $\mathrm{HZ}$ patients were lower than those in $\mathrm{HC}$ group $(P<0.05)$. In beta band, the global efficiency and local efficiency of $\mathrm{HZ}$ patients group decreased, compared with healthy group $(P<0.05)$. The functional ICA showed that three components showed significant differences between the two groups. In component 2, HZ patients showed excess superior frontal gyrus (BA10) neuro oscillation in delta band and less medial frontal gyrus (BA 11) neuro oscillation in beta and gamma bands than that in HCs. And for component 3, the alpha band of the $\mathrm{HZ}$ patients presented increased neuro activities in superior frontal gyrus (BA 11) and decreased neuro activities in occipital lobe (BA 18). In component 4, the inferior frontal gyrus (BA 47) showed excess activity in the left hemisphere and reduced activity in the right hemisphere in delta band, compared with HC group. Conclusion: Altered brain networks exist in resting-state EEG data of patients with acute HZ. The changes of EEG brain networks in HZ patients are characterized by decreased global efficiency and local efficiency in beta band. Moreover, the spontaneous oscillation of some brain regions involving pain management and the connectivity of default mode network changed in $\mathrm{HZ}$ patients. Our study provided novel understanding of $\mathrm{HZ}$ from an electrophysiological view, and led to converging evidence for treatment of $\mathrm{HZ}$ with neural regulation in future.

Keywords: herpes zoster, brain network, graph theory analysis, independent component analysis, phase locking value

\section{Introduction}

Peripheral herpes zoster (HZ) is mainly characterized by blistering dermatomal rash along the nerve and moderate to severe pain in a variety of skin, such as burning, stabbing, soreness, bloating, or allodynia. ${ }^{1}$ It is caused by the reactivation of latent 
varicella zoster virus (VZV) in spinal or cranial sensory ganglia of people who have been primary infected with $\mathrm{VZV}^{2}$ The incidence rate of $\mathrm{HZ}$ ranged 3-5/1,000 per year ${ }^{3}$ and $29.8 \%$ of $\mathrm{HZ}$ patients leave over the postherpetic neuralgia, ${ }^{4}$ which could cause negative effects on the mood and sleep quality of $\mathrm{HZ}$ patients. ${ }^{5}$

The question how pain is processed in the brain has been an enduring puzzle. A large number of studies focused on changes in pain modulation pathways and brain regions in $\mathrm{HZ}$ patients. ${ }^{6,7}$ Recently, an increasing number studies have recognized that pain may reorganize the brain networks. ${ }^{89}$ The brain network was used to describe the connectivity between interest nodes of the brain regions, ${ }^{10,11}$ which has been widely used in brain network research of neurological diseases such as epilepsy and Alzheimer's disease. ${ }^{12-16}$ Some studies using functional magnetic resonance imaging (fMRI) have found altered functional networks patients with peripheral $\mathrm{HZ}$, which may indicate that the pain processing in $\mathrm{HZ}$ patients were different from normal subjects. ${ }^{17,18}$ These studies on $\mathrm{HZ}$ are biologically meaningful considering that their findings may provide new understandings on the multiple dimensions of pain processing. However, few studies concentrated on brain function changes and pain modulation mechanisms in patients with acute $\mathrm{HZ}$ using EEG.

A widely accepted brain network analysis method: Independent component analysis (ICA) is a classical electroencephalogram (EEG) signal analysis technique, which separates scalp electrical signals into their additive independent or source components. ${ }^{19}$ All the components activated in the results are statistically independent of each other to identify independent spatial brain networks and their interactions in frequency bands. ${ }^{20}$ Another commonly used phase interaction measure is the Phase Locking Value (PLV), the absolute value of the mean phase difference between two signals from pairs of electrodes as a complex unit-length vector. ${ }^{21}$ The PLVs reflect information transfer from one brain region to other regions. ${ }^{22}$ In addition, graph theory analysis applied to the constructed PLV matrices is useful in identifying differences in brain network patterns. ${ }^{23}$ Considering that EEG signals are a complex sum of several sources of electrical brain activity, we may acquire a deeper understanding of the neurobiological mechanisms of $\mathrm{HZ}$ using these analysis methods involving brain networks. Therefore, we decided to use ICA, PLV, and graph theoretical analysis to explore the changes of brain networks in patients with acute HZ.
In the present study, we hypothesized that brain networks altered in those patients with acute HZ. Thus, we aimed to investigate whether and how EEG brain networks changed in $\mathrm{HZ}$ patients. This is practical considering that in future we may adopt novel neural regulation protocols to treat acute HZ.

\section{Materials and Methods Participants}

Seventy-one patients who were diagnosed with acute HZ from January 2016 to December 2020 in Jianyang People's Hospital were included. Inclusion criteria: 1) a diagnosis of acute herpes zoster by specialized clinicians and aged 30-80 years; 2) the onset time of acute HZ less than 14 days; 3) the patients reported no other diseases might affect their EEG patterns; 4) no previous history of nervous system disease, mental disease, or head trauma; and 5) MRI scanning on brain showed negative intracranial results. Additionally, 71 age- and sex-matched healthy control subjects who visited our hospital for routine physical examinations were enrolled as the healthy control (HC) group. No history of neurological or psychiatric disorders was reported in the controls. All included subjects were right-handed. No identifiable features for all subjects were reported in the current study. Written informed consent was obtained from the included subjects before data collection and review of the medical records. The study was approved by the Ethics Committee of the Jianyang People's Hospital. This study was conducted following the 2008 Helsinki declaration.

\section{EEG Data Acquisition}

Ten-minute scalp EEG data were collected from all included subjects. The EEG data were recorded using a 16-channel analog recorder (NT9200, Xintuo, China) continuously for ca. 10 minutes according to the international 10-20 system. The electrodes (FP1, FP2, F3, F4, F7, F8, T3, T4, T5, T6, C3, C4, P3, P4, O1, and O2) were placed using a quantified ruler. The impedance of each electrode was kept at less than $10 \mathrm{k} \Omega$. The sampling rate was $500 \mathrm{~Hz}$. During the recording time, subjects were asked to keep relaxed with their eyes closed.

\section{EEG Preprocessing}

All the original EEG data were exported as European Data Format (EDF). The EEG was bandpass filtered between 1 $\mathrm{Hz}$ and $45 \mathrm{~Hz}$ using a hamming window after the FIR filter. Then the electromyogram artefacts and muscle 
artifacts were completely removed using an EEGlab plugin-in AAR (http://germangh.com). In this tool, the blind source separation algorithm was employed to perform electrooculogram (fast ICA algorithm) corrections automatically. The EEG matrix with a low fractal dimension would be identified as electrooculogram components and would be removed automatically. ${ }^{24}$ And the blind source separation algorithm based on canonical correlation analysis also performed electromyogram (fast ICA algorithm) corrections automatically. $^{25}$

Then, the EEG was recomputed to the common average reference. The preprocessed EEG data was cut out and translated to text format for subsequent analysis. We obtained five 10-second-epochs of EEG in the resting state for each subject. The EEG data were preprocessed using EEGlab (v14.1.1, http://sccn.ucsd.edu).

\section{PLV Based Brain Network Analysis}

Electrophysiological signals can be usefully characterized in terms of their oscillatory components, through bandpass filtering into the standard frequency bands (delta, theta, alpha, beta, and gamma). Interactions can then be analyzed using measures of within and between frequency-band coupling between electrode pairs. ${ }^{26}$ Since the brain is a nonlinear dynamical system, we can use phase locking to quantify the interactions of pairs of electrodes.

The absolute value of the average phase difference between two signals is characterized by PLV, which can be used to measure the degree of synchronization of EEG signals in a certain frequency band. This method has been descripted in other studies. ${ }^{27,28}$ In brief, the PLV is defined as

$$
\operatorname{PLV}(t)=\frac{1}{\mathrm{~N}}\left|\sum_{n=1}^{N} \exp \left(j\left(\Delta \varphi_{n}(t)\right)\right)\right|
$$

where $\mathrm{N}$ denotes the total number of sampling points, and $\Delta \varphi_{n}(t)=\left(\varphi_{x}(t)-\varphi_{y}(t)\right)$ is the instantaneous difference between signal $x$ and $y$ at time $t$. The PLV takes values on $[0,1]$ with 0 reflecting the case where there is no phase synchronization and the two signals are independent and have no obvious synchronization, and 1 where the degree of synchronization between the two signals is the strongest.

In this study, seven frequency bands were defined: delta band $(1-4 \mathrm{~Hz})$, theta band $(4-8 \mathrm{~Hz})$, alphal band (8-10 Hz), alpha2 band $(10-13 \mathrm{~Hz})$, beta band (13-30 $\mathrm{Hz}$ ), gamma band (30-45 Hz), and full band (1-45 Hz).
Then, a $16 \times 16$ PLV matrix was constructed for each subject and used for later graph theory analysis.

\section{Graph Theory Analysis}

In this study, the 16 EEG channels were used as nodes for each subject in each frequency band. And the previously constructed $16 \times 16$ PLV matrix was used for graph theory analysis. We selected the path length, global efficiency, clustering coefficient, and local efficiency of the PLV network attribute for characterizing brain network topology. The path length, which referred to the average distance between any two nodes, represented the ability of the network to transmit signals. The global efficiency referred to the reciprocal average of the shortest path length, which represented the efficiency of signal transmission between network nodes and the local efficiency was the trend to measure the clustering of nodes with strong connections in a network. These graph-based indices were computed using MATLAB functions embedded in the Brain Connectivity Toolbox (http://www.brain-connectivity-toolbox.net).

\section{The Functional Independent Component Analysis (flCA)}

To analyze the differences of resting state functional network between two groups, an exact Low Resolution Electromagnetic Tomography (eLORETA) functional ICA method was employed. eLORETA is a widely used method to localize multiple distributed cortical sources of EEG data in three-dimensional space. ${ }^{29}$ The LORETA method has been previously validated in many real human EEG data. The present used eLORETA (v20190226) is an improvement over earlier related versions of LORETA $^{29}$ or Standardized LORETA (sLORETA). ${ }^{30}$ ICA is a mathematical decomposition method that separates the EEG signal into a set of statistically independent components. All the technical details can be found in other papers. ${ }^{31,32}$

In brief, first, the EEG signals were transformed to cossspectral EEG matrix for each frequency band using the discrete Fourier transform. Then, these transformed files were used for computing the spectral density for each cortical voxel and for each frequency band using the algorithm described in detail in previous literature. ${ }^{31,33}$ Thus, we obtained six eLORETA images of cortical spectral density across six frequency bands (delta band $(1-4 \mathrm{~Hz})$, theta band (4-8 Hz), alpha1 band (8-10 Hz), alpha2 band (10-13 Hz), beta band $(13-30 \mathrm{~Hz})$, gamma band $(30-45 \mathrm{~Hz}))$. These data 
correspond to a "function" of space (cortical voxel) and frequency. After that, the data from each subject in two groups is concatenated. Thus, a matrix including two dimensions was created, in which one dimension corresponds to different subjects, and the other dimension corresponds to space-frequency. This process was known as functional data analysis. When independent component analysis is used to this matrix, general networks are found. This method is defined as functional ICA. Each functional network is composed of six images, one for each frequency band. ${ }^{32}$ Those components with $Z$ scores larger than or equal to "3" were selected.

The present study was intended to achieve an optimal number of components. We tested a series number from "6" to " 16 " and found 12 components would obtain the maximum differences between two groups. Thus, the optimal number of components were defined as " 12 " in this study.

\section{Statistical Evaluations}

Measurement data were represented by mean \pm standard error of the mean (SEM). Two sample $t$-tests were used for detecting the age differences and Chi-square test was applied for comparison of gender ratio. Two sample $t$-tests with FDR correction were used for comparison of the PLV connections, network properties, and ICA components between the two groups. $P<0.05$ was considered as statistically significant.

\section{Results}

\section{Demographic results}

Seventy-one HZ patients (aged $53.56 \pm 14.58$ years) were included in this study. At the same time, 71 cases matched for age and sex served as healthy controls (aged 53.55 \pm 15.11 years). All $71 \mathrm{HZ}$ patients received acyclovir 0.5 gram Q.8H. treatment. There was no significant difference in gender and age between the two groups (Table 1).

\section{Brain Network Connectivity}

Compared with the healthy group, the connections between bilateral anterior temporal and right central regions in herpes zoster patients were decreased in beta band (Figure 1A). No significant survival different connections were found in other frequency bands after FDR corrections between the two groups (not shown).
Table I Demographics of Subjects Included in the Study

\begin{tabular}{|c|c|c|c|c|}
\hline & $\begin{array}{c}\text { HZ Group } \\
(n=7 I)\end{array}$ & $\begin{array}{c}\text { HC Group } \\
(n=71)\end{array}$ & $\chi^{2 / t}$ & $P$ \\
\hline Male:female ${ }^{\#}$ & $29 / 42$ & $32 / 39$ & 0.259 & 0.61 \\
\hline Age (years, mean $\pm S D)^{\# \#}$ & $53.56 \pm 14.58$ & $53.55 \pm 15.11$ & -0.01 & 0.99 \\
\hline Acyclovir & $7 \mid / 71$ & - & - & - \\
\hline
\end{tabular}

Note: ${ }^{\#}$ Chi-square test, ${ }^{\#}$ Two sample $t$-test.

Abbreviations: HZ, herpes zoster; HC, healthy control.

\section{Network Properties}

We computed four parameters of network properties, which were based on the constructed PLV weighted matrix, including clustering coefficient, shortest path length, global efficiency, and local efficiency. Compared with the HC group, the clustering coefficient and the local efficiency of $\mathrm{HZ}$ patients decreased in full band $(1-45 \mathrm{~Hz})$ and the clustering coefficient, the global efficiency, and the local efficiency of $\mathrm{HZ}$ patients group decreased in beta band $(13-30 \mathrm{~Hz})$. The shortest path length, however, increased in HZs in this band (Figure $1 \mathrm{~B}$ and $\mathrm{C}$ ). For other frequency bands, no significant differences were found between the two groups.

\section{ICA Networks}

Three components, numbered 2, 3, and 4, with the maximum differences between $\mathrm{HZ}$ and $\mathrm{HC}$ groups were obtained in the ICA analysis. There were greatest differences in delta, beta, and gamma band between two groups in component 2 $(P<0.05, t=2.42)$. Compared with HCs, superior frontal gyrus (SFG) (BA10) in HZs showed excessed beta oscillation, and medial frontal gyrus (BA 11) showed reduced neuro oscillation in beta and gamma bands. For component 3, compared with the $\mathrm{HC}$ group, the $\mathrm{HZ}$ patients in alpha1 and alpha2 bands showed increased activity in SFG (BA 11) and decreased activity in occipital lobe (BA 18) $(P<0.05$, $t=2.13$ ). In component 4 patients with $\mathrm{HZ}$, the inferior frontal gyrus (BA 47) showed excess activity in the left hemisphere and reduced activity in the right hemisphere in the delta band, compared with HC group. Furthermore, left middle frontal gyrus (MFG) presented excess theta activity $(P<0.05, t=2.49$ ) (Table 2). For other components, no significant differences were found between the two groups (Figure 2, Table 2).

\section{Discussion}

In the present study, we applied graph theory analysis and ICA analysis to detect the potential abnormal changes of EEG in HZ patients. We confirmed that the brain networks changed in 

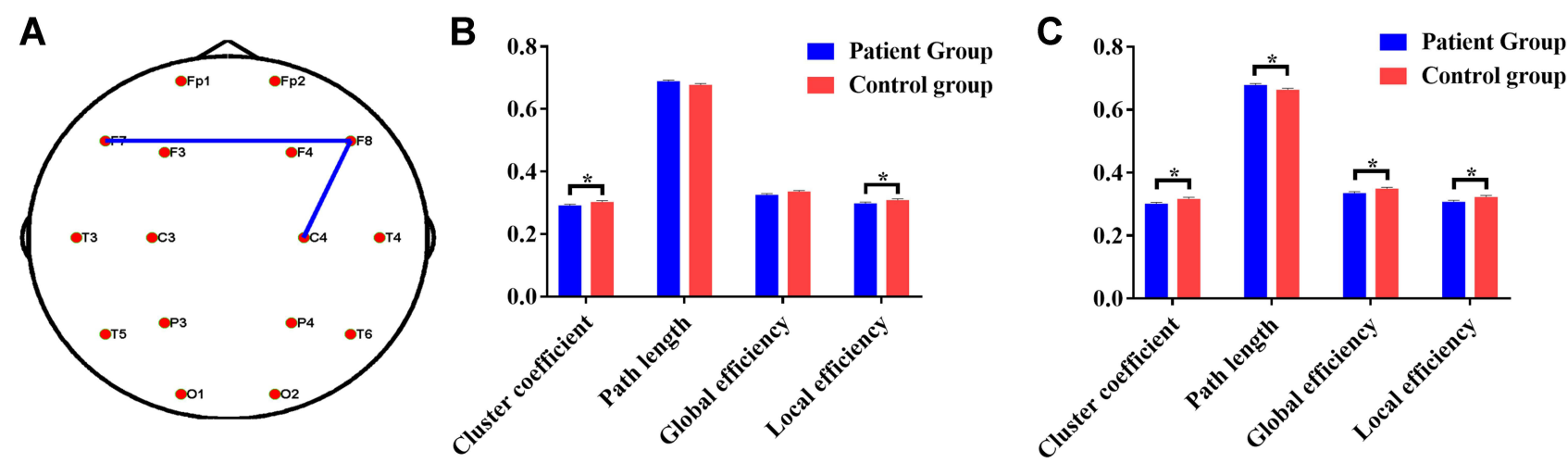

Figure I Comparisons of PLV network and graph theory properties between $\mathrm{HZ}$ and HC patients*. PLV network connectivity in beta band (I $3-30 \mathrm{~Hz}$ ) (A); Comparison of network properties in full band $(1-45 \mathrm{~Hz})(\mathbf{B})$ and beta band $(13-30 \mathrm{~Hz})(\mathbf{C})$. The blue line in $\mathrm{A}$ indicates that the connections between bilateral anterior temporal, right anterior temporal, and right central regions in $\mathrm{HZ}$ patients decreased when compared with $\mathrm{HCs}$ in beta band. No significantly different connection survival was found in other frequency bands after FDR corrections between the two groups. For B and C, the tail on each bar indicates the corresponding standard error of the mean (SEM). For other frequency bands, no significant differences were found between the two groups (not shown in the figure). *All the results were computed using two sample $t$-test and corrected with FDR method at level $P<0.05$.

patients with acute $\mathrm{HZ}$, which was characterized by decreased clustering coefficient, global efficiency, local efficiency, and increased shortest path length in the beta band. Furthermore, some brain regions involved pain management, such as SFG and MFG. These findings deepen our understanding of $\mathrm{HZ}$ from brain electrophysiological aspects.

PLV analysis showed that the synchronization of bilateral anterior temporal and right central EEG signals in $\beta$ -

Table 2 The Location Assignments of Peak Voxels for flCA*

\begin{tabular}{|c|c|c|c|c|c|c|}
\hline \multirow[t]{2}{*}{$\begin{array}{l}\text { Components and } \\
\text { Frequency Band }\end{array}$} & \multicolumn{3}{|c|}{$\begin{array}{l}\text { Peak MNI } \\
\text { Coordinates }\end{array}$} & \multirow[t]{2}{*}{ Lobe } & \multirow[t]{2}{*}{ BA } & \multirow[t]{2}{*}{ Structure } \\
\hline & $\mathbf{x}$ & $y$ & $\mathbf{z}$ & & & \\
\hline \multicolumn{7}{|l|}{ Components 2} \\
\hline Delta & 20 & 65 & -5 & Frontal & 10 & SFG \\
\hline Beta & 5 & 65 & -15 & Frontal & 11 & MFG \\
\hline Gamma & 5 & 65 & -15 & Frontal & 11 & MFG \\
\hline \multicolumn{7}{|l|}{ Components 3} \\
\hline \multirow[t]{2}{*}{ Alphal } & -15 & 65 & -15 & Frontal & 11 & SFG \\
\hline & -5 & -100 & 15 & Occipital & 18 & Cuneus \\
\hline \multirow[t]{2}{*}{ Alpha2 } & -15 & 65 & -15 & Frontal & II & SFG \\
\hline & -5 & -100 & 15 & Occipital & 18 & Cuneus \\
\hline \multicolumn{7}{|l|}{ Components 4} \\
\hline \multirow[t]{2}{*}{ Delta } & -50 & 40 & -10 & Frontal & 47 & Left IFG \\
\hline & 50 & 25 & -15 & Frontal & 47 & Right IFG \\
\hline Theta & -45 & 50 & -10 & Frontal & 11 & MFG \\
\hline
\end{tabular}

Notes: *These three components showed significant differences between $\mathrm{HZ}$ patients and the healthy group $(P<0.05)$. Other components in the two groups showed no significant differences. The macro locations of these regions are shown in Figure 2. The location assignments were performed based on eLORETA methods using LORETA KEY software in MNI space.

Abbreviations: SFG, superior frontal gyrus; MFG, medial frontal gyrus; IFG, inferior frontal gyrus. band decreased in patients with acute HZ. Previous studies have confirmed that the temporal lobe cortex is involved in the modulation of pain in the brain, ${ }^{34-36}$ and the $\beta$ oscillation in the central-parietal lobe may play a role in pain perception and processing. ${ }^{34-37}$ The decrease of interneuronal connectivity in acute $\mathrm{HZ}$ plays an important role in pain perception and modulation.

Network property analysis based on graph theory can explain the neural activity in the brain from the perspective of network and information processing. ${ }^{20}$ We noted that the acute HZ patients showed reduced global and local efficiency, clustering coefficient, and increased shortest path length in beta band. These findings from graph analysis indicated that acute HZs might show potential deficits in cognition considering that beta was involved in various cognitive functions. ${ }^{38,39}$ The change of network attribute in acute $\mathrm{HZ}$ patients might be used as a biomarker for identification of cognitive function changes. However, the changes of cognitive function in patients with herpes virus infection of the peripheral nervous system were not well recognized in clinical practice. Further studies should be designed to explore the cognitive function evolutions in patients with $\mathrm{HZ}$.

Transient thermal pain stimuli could elicit significant activity in MFG. ${ }^{40}$ In present study, however, we noted that the acute $\mathrm{HZ}$ patients showed reduced beta and gamma activity in MFG. These differences might be caused by the different nature of pain or detection methods in two studies. Moreover, the reduced beta and gamma oscillation in MFG might indicate that the MFG in $\mathrm{HZ}$ patients showed inhibition responses to $\mathrm{HZ}$ pain. Another brain region, SFG, also been reported to anticipate some types of pain, ${ }^{41,42}$ showed 
A

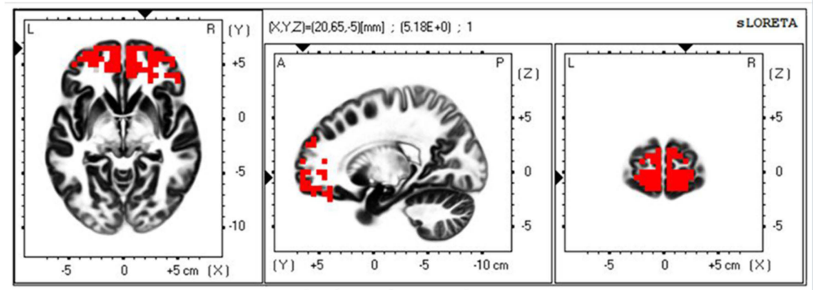

delta band component 2

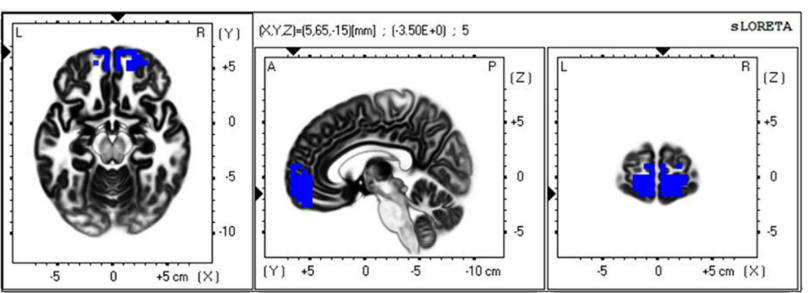

beta band

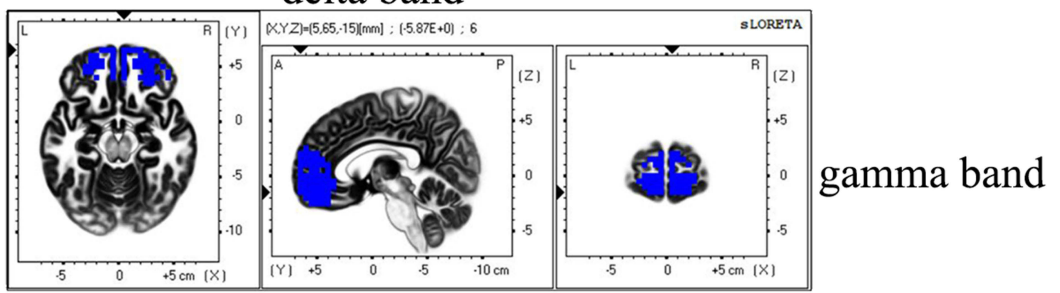

B component 3

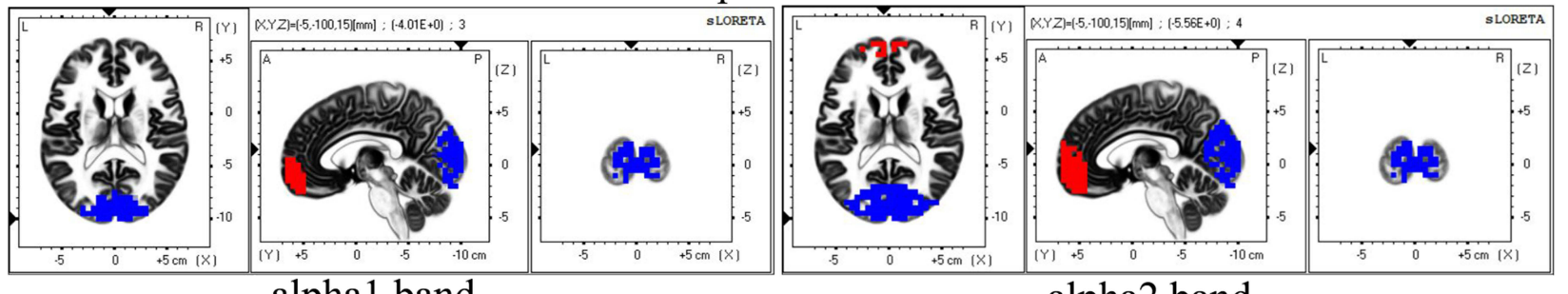

alpha1 band

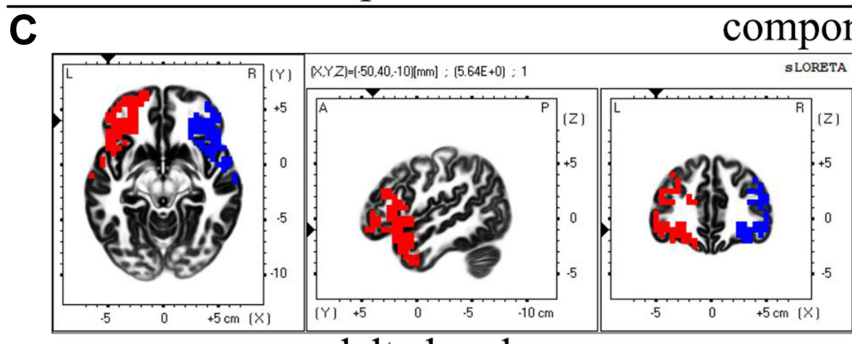

delta band

alpha2 band

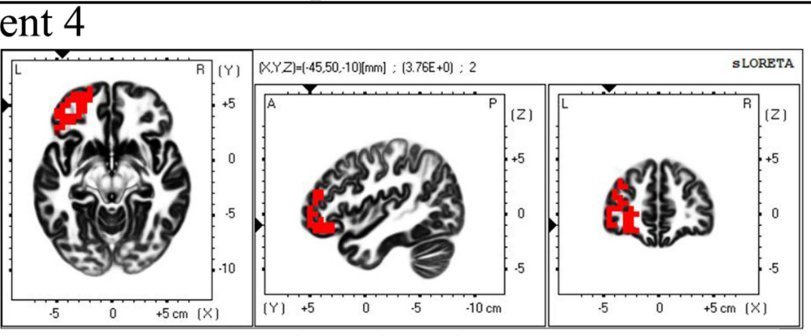

theta band

Figure 2 ICA components showing significant differences between herpes zoster patients and healthy controls. Component 2 (A), component 3 (B), and component 4 (C). The images are shown in MNI space. The red color indicates increased neuronal activity and the blue color indicates decreased neuronal activity. The small triangles along the coordinate axes indicated the maximum electric neuronal activity. The corresponding peak coordinates are displayed in the upper part of each band image. The location assignments of each component are shown in Table 2.

excessive delta and alpha activity. These findings might suggest that the balance between excitation and inhibition has altered in acute $\mathrm{HZ}$ patients.

The changes of brain electrical activity might function in network patterns in HZ. Previous fMRI studies have reported that recuperating from $\mathrm{HZ}$ accompanyies default model network (DMN) connectivity alterations. ${ }^{1}$ In the present study, it was noted that the frontal lobe anti-correlated with occipital lobe in alpha band of component 3. Parts of these regions were assignment within the range of DMN regions. This result suggested that the changes of networks in $\mathrm{HZ}$ patients involved DMN. In fact, the altered DMN has been found in multiple pain. ${ }^{43,44}$ This change might be related with pain intensity. $^{45}$
Study limitations include a relatively small sample. We did not address longitudinal effects of $\mathrm{HZ}$ on EEG, such as the associations between pain intensity and EEG networks. In addition, all patients were treated with acyclovir medication, which may have had an impact on brain networks. In future research, we will continuously collect cases and overcome these limitations for a more comprehensive understanding of $\mathrm{HZ}$ from brain electrical activity aspects.

\section{Conclusions}

Our study confirmed that the altered brain networks exist in resting-state EEG data of patients with acute HZ. The changes of EEG brain networks in $\mathrm{HZ}$ patients are characterized by decreased global efficiency and local 
efficiency in beta band. Moreover, the spontaneous oscillation of some brain regions involving pain management and the connectivity of default mode network changed in $\mathrm{HZ}$ patients. Our study provided better understanding the mechanisms of $\mathrm{HZ}$ from electrophysiological view, and led to converging evidence for treatment of $\mathrm{HZ}$ with neural regulation in future.

\section{Abbreviations}

HZ, Herpes zoster; HC, healthy control; EEG, electroencephalogram; BA, Brodmann area; ICA, Independent component analysis; eLORETA, exact Low Resolution Electromagnetic Tomography; FDR, False Discovery Rate; SFG, Superior frontal gyrus; MFG, middle frontal gyrus; DMN, default model network.

\section{Acknowledgments}

This work was supported by the Youth Program of the National Natural Science Foundation of China (81804198); Funding of Human Resources and Social Security Department, Sichuan (2018-145, 65); Youth Fund of Southwest Medical University (2018-ZRQN-003); and Research Project of Jianyang People's Hospital (JY202008). We thank the Special Training Program for Young Scientific and Technological Talents of Southwest Medical University 2020-2022 for sponsorship of Jianghai Ruan.

\section{Author Contributions}

All authors made a significant contribution to the work reported, whether that is in the conception, study design, execution, acquisition of data, analysis and interpretation, or in all these areas; took part in drafting, revising, or critically reviewing the article; gave final approval of the version to be published; have agreed on the journal to which the article has been submitted; and agree to be accountable for all aspects of the work.

\section{Disclosure}

The authors declare that they have no conflicts of interest.

\section{References}

1. Wu Y, Wang C, Qian W, et al. Disrupted default mode network dynamics in recuperative patients of herpes zoster pain. CNS Neurosci Ther. 2020;26(12):1278-1287. doi:10.1111/cns.13433

2. Schmader K. Herpes Zoster. Ann Intern Med. 2018;169(3):C19-C31. doi: $10.7326 / \mathrm{L} 18-0558$

3. Kawai K, Gebremeskel BG, Acosta CJ. Systematic review of incidence and complications of herpes zoster: towards a global perspective. BMJ Open. 2014;4(6):e4833. doi:10.1136/bmjopen2014-004833
4. Yang F, Yu S, Fan B, et al. The epidemiology of herpes zoster and postherpetic neuralgia in China: results from a cross-sectional study. Pain Ther. 2019;8(2):249-259. doi:10.1007/s40122-019-0127-z

5. Lukas K, Edte A, Bertrand I. The impact of herpes zoster and post-herpetic neuralgia on quality of life: patient-reported outcomes in six European countries. J Public Health. 2012;20(4):441-451. doi:10.1007/s10389-011-0481-8

6. Cao S, Song G, Zhang Y, et al. Abnormal local brain activity beyond the pain matrix in postherpetic neuralgia patients: a resting-state functional MRI study. Pain Physician. 2017;20(2):E303-E314. doi:10.36076/ppj.2017.e314

7. Li H, Li X, Feng Y, Gao F, Kong Y, Hu L. Deficits in ascending and descending pain modulation pathways in patients with postherpetic neuralgia. Neuroimage. 2020;221:117186. doi:10.1016/j. neuroimage.2020.117186

8. Zheng W, Woo C, Yao Z, et al. Pain-evoked reorganization in functional brain networks. Cereb Cortex. 2019;30(5):2804-2822. doi:10.1093/cercor/bhz276

9. Shen W, Tu Y, Gollub RL, et al. Visual network alterations in brain functional connectivity in chronic low back pain: a resting state functional connectivity and machine learning study. NeuroImage Clin. 2019;22:101775. doi:10.1016/j.nicl.2019.101775

10. Dennis EL, Thompson PM. Functional brain connectivity using fMRI in aging and Alzheimer's disease. Neuropsychol Rev. 2014;24 (1):49-62. doi:10.1007/s11065-014-9249-6

11. Vecchio F, Miraglia F, Maria RP. Connectome: graph theory application in functional brain network architecture. Clin Neurophysiol Pract. 2017;2:206-213. doi:10.1016/j.cnp.2017.09.003

12. Mazrooyisebdani M, Nair VA, Garcia-Ramos C, et al. Graph theory analysis of functional connectivity combined with machine learning approaches demonstrates widespread network differences and predicts clinical variables in temporal lobe epilepsy. Brain Connect. 2020;10(1):39-50. doi:10.1089/brain.2019.0702

13. Jiang W, Li J, Chen X, Ye W, Zheng J. Disrupted structural and functional networks and their correlation with alertness in right temporal lobe epilepsy: a graph theory study. Front Neurol. 2017;8:179. doi:10.3389/fneur.2017.00179

14. Zhu J, Xu C, Zhang X, et al. The changes in the topological properties of brain structural network based on diffusion tensor imaging in pediatric epilepsy patients with vagus nerve stimulators: a graph theoretical analysis. Brain Dev. 2021;43(1):97-105. doi:10.1016/j. braindev.2020.07.006

15. Behfar Q, Behfar SK, von Reutern B, et al. Graph theory analysis reveals resting-state compensatory mechanisms in healthy aging and prodromal Alzheimer's disease. Front Aging Neurosci. 2020;12:576627. doi:10.3389/fnagi.2020.576627

16. Franciotti R, Falasca NW, Arnaldi D, et al. Cortical network topology in prodromal and mild dementia due to Alzheimer's disease: graph theory applied to resting state EEG. Brain Topogr. 2019;32 (1):127-141. doi:10.1007/s10548-018-0674-3

17. Wu Y, Wang C, Yu L, et al. Abnormal within- and cross-networks functional connectivity in different outcomes of herpes zoster patients. Brain Imaging Behav. 2021. doi:10.1007/s11682-021-00510-y

18. Tang Y, Ren C, Wang M, et al. Altered gray matter volume and functional connectivity in patients with herpes zoster and postherpetic neuralgia. Brain Res. 2021;1769:147608. doi:10.1016/j.brainres.2021.147608

19. Zhang W, Shi Z, Tang H, Tang Y. [Blind source separation for fMRI signals using a new independent component analysis algorithm and principal component analysis]. Sheng Wu Yi Xue Gong Cheng Xue Za Zhi. 2007;24(2):430-433. Chinese.

20. Xiao F, Lu C, Zhao D, et al. Independent component analysis and graph theoretical analysis in patients with narcolepsy. Neurosci Bull. 2019;35(4):743-755. doi:10.1007/s12264-018-0307-6

21. Lachaux J, Rodriguez E, Martinerie J, Varela FJ. Measuring phase synchrony in brain signals. Hum Brain Mapp. 1999;8(4):194-208. doi:10.1002/(SICI)1097-0193(1999)8:4<194::AID-HBM4>3.0.CO;2-C 
22. Kamijo K. Chapter 10 - Physical activity, fitness, and cognition: insights from neuroelectric studies. In: McMorris T, editor. ExerciseCognition Interaction. San Diego: Academic Press; 2016:211-226.

23. Vecchio F, Miraglia F, Alu F, et al. Contribution of graph theory applied to EEG data analysis for Alzheimer's disease versus vascular dementia diagnosis. $J$ Alzheimers Dis. 2021;82(2):871-879. doi:10.3233/JAD-210394

24. Gómez-Herrero G, De Clercq W, Anwar H, et al. Automatic removal of ocular artifacts in the EEG without an EOG reference channel. Paper presented at: Proceedings of the 7th Nordic Signal Processing Symposium - NORSIG; 2006; 0007-09-20, 2006.

25. De Clercq W, Vergult A, Vanrumste B, Van Paesschen W, Van Huffel S. Canonical correlation analysis applied to remove muscle artifacts from the electroencephalogram. IEEE Trans Biomed Eng. 2006;53(12):2583-2587. doi:10.1109/TBME.2006.879459

26. Aydore S, Pantazis D, Leahy RM. A note on the phase locking value and its properties. Neuroimage. 2013;74:231-244. doi:10.1016/j. neuroimage.2013.02.008

27. Bandarabadi M, Gast H, Rummel C, et al. Assessing epileptogenicity using phase-locked high frequency oscillations: a systematic comparison of methods. Front Neurol. 2019;10:1132. doi:10.3389/ fneur.2019.01132

28. Chikara RK, Lo WC, Ko LW. Exploration of brain connectivity during human inhibitory control using inter-trial coherence. Sensors. 2020;20(6):1722. doi:10.3390/s20061722

29. Pascual-Marqui RD, Michel CM, Lehmann D. Low resolution electromagnetic tomography: a new method for localizing electrical activity in the brain. Int $J$ Psychophysiol. 1994;18(1):49-65. doi:10.1016/0167-8760(84)90014-X

30. Pascual-Marqui RD. Standardized low-resolution brain electromagnetic tomography (sLORETA): technical details. Methods Find Exp Clin Pharmacol. 2002;24(Suppl D):5-12.

31. Pascual-Marqui R, Kochi K, Lehmann D, Koukkou M, Kinoshita T. Functional independent components: revealing cortico-cortical, cross-frequency interactions. Jpn $J$ Pharmacol EEG. 2011;12:53-58. doi:10.5167/uzh-48682

32. Aoki Y, Ishii R, Pascual-Marqui RD, et al. Detection of EEG-resting state independent networks by eLORETA-ICA method. Front Hum Neurosci. 2015;9:31. doi:10.3389/fnhum.2015.00031

33. Pascual-Marqui RD, Biscay-Lirio RJ. Interaction patterns of brain activity across space, time and frequency. Part I: methods. arXiv Preprint arXiv:1103 2852; 2011.
34. Weissman-Fogel I, Moayedi M, Tenenbaum HC, Goldberg MB, Freeman BV, Davis KD. Abnormal cortical activity in patients with temporomandibular disorder evoked by cognitive and emotional tasks. Pain. 2011;152(2):384-396. doi:10.1016/j.pain.2010.10.046

35. Schmidt-Wilcke T, Hierlmeier S, Leinisch E. Altered regional brain morphology in patients with chronic facial pain. Headache. 2010;50 (8):1278-1285. doi:10.1111/j.1526-4610.2010.01637.x

36. Ploner M, Lee MC, Wiech K, Bingel U, Tracey I. Flexible cerebral connectivity patterns subserve contextual modulations of pain. Cereb Cortex. 2011;21(3):719-726. doi:10.1093/cercor/bhq146

37. Wei M, Liao Y, Liu J, et al. EEG beta-band spectral entropy can predict the effect of drug treatment on pain in patients with herpes zoster. $J$ Clin Neurophysiol. 2020. doi:10.1097/WNP.0000000000000758

38. Schmidt R, Herrojo Ruiz M, Kilavik BE, Lundqvist M, Starr PA, Aron AR. Beta oscillations in working memory, executive control of movement and thought, and sensorimotor function. J Neurosci. 2019;39(42):8231-8238. doi:10.1523/JNEUROSCI.1163-19.2019

39. Moriarty O, McGuire BE, Finn DP. The effect of pain on cognitive function: a review of clinical and preclinical research. Prog Neurobiol. 2011;93(3):385-404. doi:10.1016/j.pneurobio.2011.01.002

40. Brooks JC, Nurmikko TJ, Bimson WE, Singh KD, Roberts N. fMRI of thermal pain: effects of stimulus laterality and attention. Neuroimage. 2002;15(2):293-301. doi:10.1006/nimg.2001.0974

41. Choi JC, Park SK, Kim YH, et al. Different brain activation patterns to pain and pain-related unpleasantness during the menstrual cycle. Anesthesiology. 2006;105(1):120-127. doi:10.1097/00000542200607000-00021

42. Symonds LL, Gordon NS, Bixby JC, Mande MM. Right-lateralized pain processing in the human cortex: an FMRI study. $J$ Neurophysiol. 2006;95(6):3823-3830. doi:10.1152/jn.01162.2005

43. Alshelh Z, Marciszewski KK, Akhter R, et al. Disruption of default mode network dynamics in acute and chronic pain states. Neuroimage Clin. 2018;17:222-231. doi:10.1016/j.nicl.2017.10.019

44. Loggia ML, Kim J, Gollub RL, et al. Default mode network connectivity encodes clinical pain: an arterial spin labeling study. Pain. 2013;154(1):24-33. doi:10.1016/j.pain.2012.07.029

45. Jones SA, Morales AM, Holley AL, Wilson AC, Nagel BJ. Default mode network connectivity is related to pain frequency and intensity in adolescents. NeuroImage Clin. 2020;27:102326. doi:10.1016/j. nicl.2020.102326
Journal of Pain Research

\section{Publish your work in this journal}

The Journal of Pain Research is an international, peer reviewed, open access, online journal that welcomes laboratory and clinical findings in the fields of pain research and the prevention and management of pain. Original research, reviews, symposium reports, hypothesis formation and commentaries are all considered for publication. The manuscript management system is completely online and includes a very quick and fair peer-review system, which is all easy to use. Visit http:// www.dovepress.com/testimonials.php to read real quotes from published authors. 